\title{
Thermodynamics of localized magnetic moments in a Dirac conductor
}

\author{
V. Cheianov, M. Szyniszewski, E. Burovski, Yu. Sherkunov, and V. Fal'ko \\ Physics Department, Lancaster University, Lancaster LAI 4YB, England, United Kingdom
}

(Received 17 December 2011; published 16 August 2012)

\begin{abstract}
We show that the magnetic susceptibility of a dilute ensemble of magnetic impurities in a conductor with a relativistic electronic spectrum is nonanalytic in the inverse temperature at $T^{-1} \rightarrow 0$. We derive a general theory of this effect and construct the high-temperature expansion for the disorder averaged susceptibility to any order, convergent at all temperatures down to a possible ordering transition. When applied to Ising impurities on a surface of a topological insulator, the proposed general theory agrees with Monte Carlo simulations, and it allows us to find the critical temperature of the ferromagnetic phase transition.
\end{abstract}

DOI: 10.1103/PhysRevB.86.054424

\section{INTRODUCTION}

Collective phenomena in random ensembles of magnetic impurities embedded in metallic conductors are caused by the long-distance exchange interaction mediated by the mobile carriers, also known as the Ruderman-Kittel-Kasuya-Yosida (RKKY) exchange. ${ }^{1}$ The RKKY interaction has a wellstudied universal structure for all metallic systems, ${ }^{2}$ with the exception of the recently discovered class of two-dimensional (2D) materials in which the low-energy electron excitations resemble massless Dirac particles: graphene, ${ }^{3,4}$ chiral metals formed at the surface of topological insulators, ${ }^{5,6}$ and silicene. ${ }^{7}$ Recent experiments ${ }^{8,9}$ have reported the formation of a band gap in a chiral metal contaminated by magnetic impurities, pointing towards magnetic ordering at the surface of the topological insulator; also theoretical modeling suggested ordering transitions in some such systems. ${ }^{10,11}$

There are two peculiarities of the RKKY exchange in planar conductors with the Dirac-like electron spectrum which make it qualitatively different from usual metals: (i) the exchange interaction as a function of distance between two impurities shows the unusual $1 / r^{3}$ decay law and (ii) the Friedel oscillations are either absent or commensurate with the lattice. ${ }^{12}$ In the following, we shall call such interaction a Dirac-RKKY exchange. Other details of the RKKY exchange such as its anisotropy or whether it is ferromagnetic, antiferromagnetic, or depolarizing may depend on the material and the symmetry of the impurity position in the lattice. In this work we propose a general quantitative theory of the thermodynamics of an ensemble of randomly positioned magnetic impurities interacting through the Dirac-RKKY exchange in a paramagnetic phase, that is at temperatures above the magnetic ordering temperature $T_{c}$. We show that, due to a peculiar decay law of the Dirac-RKKY exchange, the magnetic susceptibility shows a strong deviation from the Curie-Weiss law seen as nonanaliticity in its high-temperature expansion:

$$
\chi=\frac{c_{0}}{T} \sum_{n=1}^{\infty} C_{n}\left(\frac{T_{0}}{T}\right)^{\frac{2}{3}(n-1)},
$$

where $c_{0}$ is the Curie constant, $T_{0} \propto \rho^{3 / 2}$ is a temperature scale dependent on the density of impurities $\rho$, and $C_{n}$ are numerical coefficients expressed in terms of finite-dimensional integrals of elementary functions [see Eqs. (6) and (7)]. The expansion Eq. (1) also encodes detailed information about the critical point of a magnetic transition: the value of $T_{c}$ and the susceptibility critical exponent $\gamma$ can be extracted from the values of several coefficients $C_{n}$ with the help of the Padé approximation. ${ }^{13}$ One example of a successful application of the proposed theory is illustrated in Fig. 1, where the susceptibility obtained with the help of Eq. (1) is compared with the Monte Carlo data for the archetypal Dirac-RKKY system of randomly positioned Ising spins with ferromagnetic $1 / r^{3}$ exchange. Numerical values of the coefficient $C_{2}$ for some other Dirac-RKKY models are presented in Table I.

A pair of localized spins separated by a distance exceeding a few lattice constants experiences the RKKY interaction mediated by electrons near the Fermi surface, which in Dirac conductors consists of a discrete set of points in the reciprocal space. In graphene there are two Fermi points related by time-reversal symmetry. ${ }^{4}$ In topological insulators where the ultrarelativistic electrons reside at the surface, there may be one Fermi point per face. ${ }^{5}$ The dispersion relation of the electronic excitations near each Fermi point is $\epsilon(p)= \pm \hbar v p$, where $p$ is the Bloch wave number of an electron relative to the Fermi point. Due to the discrete geometry of the Fermi surface and the linear dispersion of the excitations, the Dirac-RKKY interaction does not exhibit Friedel oscillations found in usual metals and decays as $1 / r^{3}$ as a function of distance $r$ between spins. ${ }^{10,14,15}$ This decay law is valid in a broad range of lengths $r_{E}<r<r_{T}$, where $r_{E}=\hbar v / E$ is the length scale associated with the binding energy $E(\sim 1 \mathrm{eV})$ of adatom, and $r_{T}=\hbar v / k T$ is the thermal wavelength of an electron. The most general form of the Dirac-RKKY Hamiltonian of a system of $N$ impurities in a magnetic field is

$$
\begin{aligned}
H_{12 \ldots N} & =J \sum_{i<j}^{N} \frac{G_{i j}}{r_{i j}^{3}}-h S_{z}, \\
G_{i j} & =G\left(\mathbf{s}_{i}, \mathbf{s}_{j}, \mathbf{n}_{i j}, \xi_{i}, \xi_{j}\right) .
\end{aligned}
$$

Here the sum is taken over all pairs of impurities randomly distributed with the average density $\rho$ in the conductor plane, and $r_{i j}=\left|\mathbf{r}_{i}-\mathbf{r}_{j}\right|$ is the distance between two impurities with quenched positions $\mathbf{r}_{i}$ and $\mathbf{r}_{j}$. We assume that there are no spatial correlations in impurities' positions. This limits our analysis to temperatures $T<J / r_{I}^{3}$, where $r_{I}$ is the correlation radius in the impurity distribution. For neutral adatoms we expect $r_{I} \approx r_{E}$. The magnetic field $h$ in Eq. (2) is coupled to the $z$ projection of the total spin $S_{z}=s_{1}^{z}+\ldots s_{N}^{z}$, and the spin of each impurity is assumed to be in the $2 s+1$ 


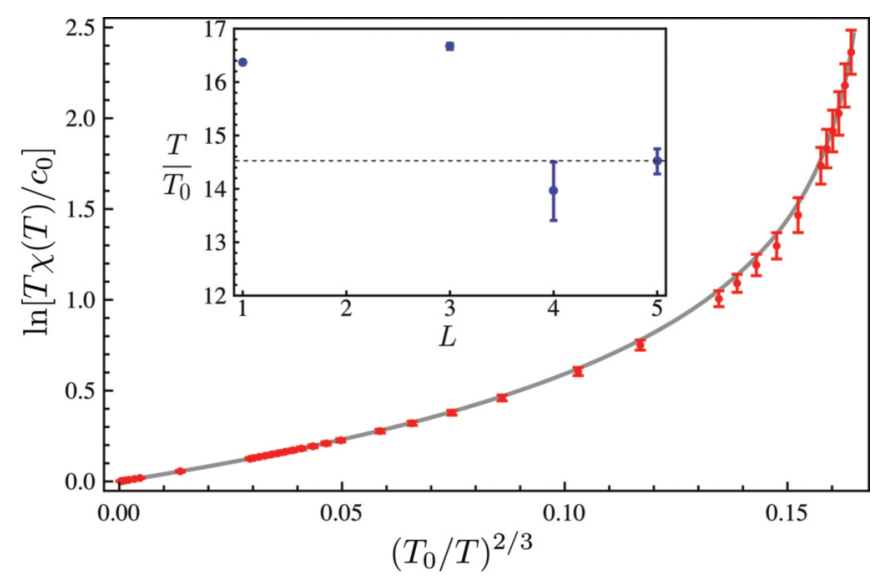

FIG. 1. (Color online) Magnetic susceptibility of the DiracRKKY Ising model. The solid line shows the $L=5$ D-log Padé approximant calculated from the first twelve terms in the expansion Eq. (1) (see Table II for the numerical values of the coefficients $C_{n}$ ). The points with error bars are the results of the Monte Carlo simulation. The error bars show the statistical uncertainty arising from both MC fluctuations and quenched disorder. Inset: the approximate critical temperature derived from the position of the singularity of the D-log Padé approximant. The error bars show the uncertainty due to the finite precision of the expansion coefficients in Table II. The thin dotted line shows the best approximation for the critical temperature.

dimensional representation of SU(2). The parameter $J$, having the dimensions of energy times volume, is specific for the given host material and the type of impurity. Together with the impurity density $\rho$ it defines the energy scale:

$$
T_{0}=\frac{s(s+1) J}{3} \rho^{3 / 2} .
$$

Further material-dependent details of the RKKY interaction are encoded in the dimensionless pairwise interaction function $G_{i j}$, which depends on two impurity spins $\mathbf{s}_{i}, \mathbf{s}_{j}$, a unit vector $\mathbf{n}_{i j}=\mathbf{r}_{i j} / r_{i j}$, and two discrete quenched random variables $\xi_{i}, \xi_{j}$ defining the position of each adatom inside the lattice unit cell ${ }^{10,15,16}$ (see Table I for examples).

\section{NONANALITICITY OF THE HIGH-TEMPERATURE EXPANSION}

The nonanaliticity of the high-temperature expansion, Eq. (1), results from the interplay between the peculiar
$1 / r^{3}$ Dirac-RKKY interaction and the randomness of the distribution of impurities in the system. The $1 / r^{3}$ dependence of the Dirac-RKKY interaction in Eq. (2) makes it impossible to use the standard $1 / T$ expansion to analyze the susceptibility. Indeed, for any temperature $T_{c}<T<T_{E}$ the ensemble contains a finite fraction of pairs, in which the spins are close enough to each other to be strongly correlated. Consider, for example, the classical ferromagnetic Ising model with $G_{i j}=$ $-s_{i} s_{j}$, where $s_{i}= \pm s$. Due to the presence of correlated pairs the high-temperature asymptotics of the susceptibility splits into two contributions. Those spins that belong to pairs smaller than the correlation radius $R_{0}=(J / T)^{1 / 3}=\rho^{-1 / 2}\left(T_{0} / T\right)^{1 / 3}$ are strongly bound into one block spin having the length $2 s$. The fraction of such spins is $p \sim \rho R_{0}^{2}=\left(T_{0} / T\right)^{2 / 3}$. The rest of the spins are separated by distances exceeding $R_{0}$ and can be regarded as an ideal gas of spins of length $s$. Then the mixture of the ideal gas of pairs and the ideal gas of single spins has the Curie susceptibility $\chi=c_{0}(1-p) s^{2} / T+c_{0} p(2 s)^{2} / 2 T$. This expression deviates from the ideal gas susceptibility, $c_{0} s^{2} / T$, by a nonanalytic correction $\delta \chi \propto p / T \propto(T)^{-5 / 3}$ in agreement with the unusual structure of the expansion Eq. (1). It is straightforward to generalize this argument to other powers in the decay law of exchange interaction in Eq. (2). For the $1 / r^{p}$ decay law and $p>2$ the first subleading correction to the susceptibility will be proportional to $T^{-1-2 / p}$. At $p \leqslant 2$ the temperature-dependent susceptibility will be dominated by the collective long-range physics which is beyond the grasp of the present simple picture.

\section{QUANTITATIVE THEORY FOR THE DIRAC-RKKY SYSTEMS IN PARAMAGNETIC PHASE}

A quantitative theory for the Dirac-RKKY systems in paramagnetic phase requires a resummation of the shortdistance singularities appearing in the disorder average of the observables. This is achieved by combining the replica method with the virial expansion of the free energy in the temperaturedependent gas parameter $p$. Thermodynamic properties of the system are encoded in the potential

$$
F(h, T, N ; q)=-T \ln \overline{[Z(h, T, N)]^{q}},
$$

where $Z(h, T, N)$ is the partition function of a given realization of the system of $N$ impurities at temperature $T$ and in the presence of the magnetic field $h$. The integer $q$ is the number of identical replicas of the disordered system. The overline

TABLE I. Examples of Dirac-RKKY systems and the corresponding values of the coefficient $C_{2}\left(C_{1}=1\right.$ for $\left.s=1 / 2\right)$ and the generalized Curie temperature $\Theta$. The host material is assumed to be in the $x-y$ plane. The susceptibility is calculated for the observable given in the third column. The observable $\Phi$ in the last row defines a staggered order in which impurity spins in graphene are correlated with the $\sqrt{3} \times \sqrt{3}$

\begin{tabular}{|c|c|c|c|c|}
\hline Dirac-RKKY ensemble & $G_{i j}$ & Observable & $C_{2}$ & $\Theta / T_{0}$ \\
\hline Spin-1/2 Ising impurities & $-s_{i}^{z} s_{j}^{z}$ & $S_{z}$ & 9.02 & 27.1 \\
\hline Spin-1/2 impurities isotropically coupled to & $-s_{i}^{z} s_{j}^{z}-\left(\mathbf{n} \cdot \mathbf{s}_{i}\right)\left(\mathbf{n} \cdot \mathbf{s}_{j}\right)+\frac{1}{2}\left(\mathbf{n} \times \mathbf{s}_{i}\right)_{z}\left(\mathbf{n} \times \mathbf{s}_{j}\right)_{z}$ & $S_{z}$ & 9.59 & 29.7 \\
\hline the electrons in a chiral metal & & $S_{x}$ & 1.18 & 1.28 \\
\hline Spin-1/2 impurities with $\mathrm{X}-\mathrm{Y}$ coupling to & $-\left(\mathbf{n} \cdot \mathbf{s}_{i}\right)\left(\mathbf{n} \cdot \mathbf{s}_{j}\right)+\frac{1}{2}\left(\mathbf{n} \times \mathbf{s}_{i}\right)_{z}\left(\mathbf{n} \times \mathbf{s}_{j}\right)_{z}$ & $S_{z}$ & 3.17 & 5.63 \\
\hline the electrons in a chiral metal & & $S_{x}$ & 1.17 & 2.16 \\
\hline Spin-1/2 impurities in graphene located at centers of hexagons & $-\left(\mathbf{s}_{i} \cdot \mathbf{s}_{j}\right) \cos \frac{2 \pi}{3}\left(\xi_{i}-\xi_{j}\right), \quad \xi \in\{0,1,2\}$ & $\begin{array}{l}S_{z} \\
\Phi\end{array}$ & $\begin{array}{r}-2.20 \\
3.90\end{array}$ & $\begin{array}{r}-3.27 \\
7.69\end{array}$ \\
\hline
\end{tabular}
superlattice induced by the commensurate Friedel oscillations ${ }^{10,20}$. 
stands for the averaging over all quenched variables:

$$
\bar{f} \equiv \frac{1}{\Xi^{N} \mathcal{A}^{N}} \sum_{\xi_{1} \ldots \xi_{N}} \int \prod_{i=1}^{N}\left[d \mathbf{r}_{i}\right] f\left(\mathbf{r}_{1}, \ldots, \mathbf{r}_{N}, \xi_{1}, \ldots, \xi_{N}\right),
$$

where $\mathcal{A}$ is the area of the sample, and $\Xi=\sum_{\xi} 1$ is the number of distinct values of the variable $\xi$.

The magnetic susceptibility can be written as

$$
\chi(T)=\frac{c_{0}}{N} \lim _{h \rightarrow 0} \lim _{q \rightarrow 0} \frac{\partial^{2}}{\partial h^{2}} \frac{\partial}{\partial q} F(h, T, N ; q),
$$

which requires analytic continuation of the potential $F$ to the positive real axis of $q$. In order to obtain the virial expansion for the susceptibility Eq. (5) it is convenient to consider the grand canonical ensemble and introduce the thermodynamic potential

$$
\Omega=-T \ln \left[\sum_{N=0}^{\infty} \frac{e^{\frac{\mu N}{T}}}{N !} \overline{Z^{q}(h, T, N)}\right]=-T \sum_{n=1}^{\infty} \frac{V_{n}}{n !} e^{\frac{\mu n}{T}},
$$

which is related to the potential Eq. (4) by the Legendre transformation $F=\Omega+\mu N$, where $N=-\partial \Omega / \partial \mu$. The chemical potential $\mu$ is a $q$-dependent auxiliary variable, which does not have any straightforward physical meaning. The coefficients $V_{n}$ in $\Omega$ are called the virial coefficients. The first three of those are

$$
\begin{aligned}
V_{1} & =\overline{Z_{1}^{q},} \\
V_{2} & =\overline{Z_{12}^{q}-Z_{1}^{q} Z_{2}^{q},} \\
V_{3} & =\overline{\hat{S}\left[Z_{123}^{q}-3 Z_{12}^{q} Z_{3}^{q}+2 Z_{1}^{q} Z_{2}^{q} Z_{3}^{q}\right],}
\end{aligned}
$$

where $Z_{i_{1} \ldots i_{n}}=\operatorname{Tr} e^{-\beta H_{i_{1} \ldots i_{n}}}$ is a function of $n$ quenched variables $\left\{\mathbf{r}_{i_{1}}, \ldots, \mathbf{r}_{i_{n}}\right\}$ and $\left\{\xi_{i_{1}}, \ldots, \xi_{i_{n}}\right\}$ describing localized spins with indices $i_{1}, \ldots, i_{n} \in\{1, \ldots, N\}$, and $H_{i_{1}, \ldots, i_{n}}$ is the Hamiltonian Eq. (2) constrained to this $n$ subset. The trace is taken over all spin variables of the $n$ subset, and the symbol $\hat{S}$ represents a complete symmetrization of the expression over the particle indices.

After substituting the virial expansion of $\Omega$ into Eq. (5) one arrives at Eq. (1), with

$$
\begin{aligned}
C_{n}= & {\left[\frac{3}{s(s+1)}\right]^{1+\frac{2}{3}(n-1)} \frac{1}{\left[\sum_{\xi_{1}, \ldots \xi_{n}} 1\right] n !} } \\
& \times\left.\sum_{\xi_{1}, \ldots \xi_{n}} \int d \mathbf{x}_{2} \ldots d \mathbf{x}_{n} \hat{S} \frac{d^{n}}{d z^{n}} Q(z)\right|_{z=0},
\end{aligned}
$$

where the integration is over $n-1$ dimensionless variables $\left(\mathbf{x}_{2}, \ldots \mathbf{x}_{n}\right)=\left(\mathbf{r}_{2}, \ldots, \mathbf{r}_{n}\right) / R_{0}$. The generating function $Q(z)$ in Eq. (6) is defined by the infinite series

$$
\begin{aligned}
Q(z) & =e^{-z}\left(z M_{1}+\frac{z^{2}}{2 !} M_{12}+\frac{z^{3}}{3 !} M_{123}+\ldots\right), \\
M_{1 \ldots n} & =\frac{\operatorname{Tr}\left(s_{1}^{z}+\ldots+s_{n}^{z}\right)^{2} e^{-\sum_{i<j} \frac{G_{i j}}{\left|x_{i}-x_{j}\right|^{3}}}}{\operatorname{Tr} e^{-\sum_{i<j} \frac{G_{i j}}{\left|x_{i}-x_{j}\right|^{3}}}} .
\end{aligned}
$$

Note that there is no integration over $\mathbf{x}_{1}$ in Eq. (6). Equations (1), (6), and (7) constitute the main result of this work, which can be applied to any random Dirac-RKKY model.

The unusual power of $1 / T$ in the high-temperature expansion Eq. (1) is directly related to the power 3 in the decay law of the exchange interaction. One can consider a generalization of the Hamiltonian Eq. (2)

\section{HIGH-TEMPERATURE EXPANSION AND THE CRITICAL POINT OF THE ISING MODEL WITH RANDOM DIRAC-RKKY EXCHANGE}

As an illustration, we consider the paramagnetic susceptibility of the ferromagnetic Ising model described by the pairwise interaction function $G_{i j}=-s_{i}^{z} s_{j}^{z}$, where $s_{i}^{z}= \pm 1 / 2$ is the Ising spin of the $i$ th atom. This model describes, for example, an ensemble of magnetic impurities with strong outof plane exchange on the surface of a topological insulator: ${ }^{11}$ it also describes structural phase transitions in graphene. ${ }^{10}$ The only random quenched variables in this model are the positions of impurities in the sample. The first two virial coefficients found from Eqs. (6) and (7) are

$$
\begin{aligned}
& C_{1}=1, \\
& C_{2}=\frac{1}{2} \int_{0}^{\infty}\left[\frac{4}{1+e^{-\frac{2}{x^{3}}}}-2\right] 2 \pi x d x=9.01845 \ldots
\end{aligned}
$$

Similarly, all higher-order $C_{n}$ are expressed as integrals of elementary functions of increasing complexity. The numerical values of the first twelve coefficients $C_{n}$ are given inxbrk Table II.

At low enough temperature the Ising model with a ferromagnetic exchange undergoes a phase transition into a ferromagnetically ordered state. In order to extract the properties of the critical point from the high-temperature data in Table II we use the D-log Padé technique ${ }^{13}$ to investigate the function $f(w)=\sum_{n \geqslant 1} C_{n} w^{n-1}$, where $C_{n}$ are the same coefficients as appear in Eq. (1), and $w=\left(T_{0} / T\right)^{2 / 3}$. More precisely, we construct the first few $[L / L+1]$ Padé approximants, $0<L \leqslant 5$, to the function $g(w)=f^{\prime}(w) / f(w)$ and analyze the structure of the singularity of the approximants in the vicinity of the hypothetical critical point. For the

TABLE II. Numerical values of the coefficients in the high-temperature expansion Eq. (1) for the susceptibility of the ferromagnetic Ising model.

\begin{tabular}{cccccc}
\hline \hline$C_{n}$ & numerical value & $C_{n}$ & numerical value & $C_{n}$ & numerical value \\
\hline$C_{1}$ & 1 & $C_{5}$ & $3.128(2) \times 10^{3}$ & $C_{9}$ & $5.612(4) \times 10^{6}$ \\
$C_{2}$ & $9.0184534 \ldots$ & $C_{6}$ & $2.103(1) \times 10^{4}$ & $C_{10}$ & $3.328(3) \times 10^{7}$ \\
$C_{3}$ & $6.578(2) \times 10^{1}$ & $C_{7}$ & $1.3937(5) \times 10^{5}$ & $C_{11}$ & $1.857(3) \times 10^{8}$ \\
$C_{4}$ & $4.582(1) \times 10^{2}$ & $C_{8}$ & $9.007(4) \times 10^{5}$ & $C_{12}$ & $1.048(7) \times 10^{9}$ \\
\hline \hline
\end{tabular}


$L=5$ approximant, which requires the knowledge of the first twelve coefficients $C_{n}$, we solve the differential equation $d \ln [f(w)] / d w=[L / L+1]_{g}(w)$ with the initial condition $f(0)=0$ and compare the result with the susceptibility obtained from Monte Carlo simulations on an ensemble of $10^{5}$ impurities averaged over 100 configurations of quenched disorder (see Fig. 1). The simulations are performed with the classical worm algorithm ${ }^{17}$ using the Algorithms and Libraries for Physics Simulations (ALPS) ${ }^{18}$ Since the numerical values of $C_{n}$ have been calculated with finite precision, the position of the singularity of each approximant is found with some uncertainty (see the inset of Fig. 1). For the $L=5$ approximant we find $T_{c} / T_{0}=14.5(5)$, which is close to the value found from numerical simulations in Refs. 10,11,19. We also extract the value of the susceptibility critical exponent $\gamma$ by calculating the residue of the $L=5$ Padé approximant at the critical point and find that $\gamma=1.4(2)$.

\section{CONCLUSIONS}

We conclude this report with the discussion of several other examples of the Dirac-RKKY systems identified in earlier literature. A detailed analysis of criticality in such systems is beyond the scope of this work, however there is enough interesting information already contained in the leading coefficients $C_{1}$ and $C_{2}$ of the expansion Eq. (1). The ratio of these coefficients defines a a generalized Curie temperature $\Theta=\operatorname{sign}\left(C_{2}\right) \times\left|C_{2} / C_{1}\right|^{3 / 2} T_{0}$, which encodes the experimentally measurable deviation of $\chi(T)$ from the Curie law and gives an estimate of $T_{c}$ if the susceptibility is calculated for the observable corresponding to the order parameter. For spin systems having competing orders one can compare the generalized Curie temperatures extracted from the susceptibilities for the suspected order parameters for which ordering is more likely to occur. Such information is given in Table I. The first two rows describe two limiting cases of a more general model derived in Ref. 11 for the RKKY exchange in chiral metals. The authors of Ref. 11 observe that a strongly anisotropic in-plane exchange tends to destroy the magnetic order enforced by the out-of-plane ferromagnetic coupling. They also conjecture that as a function of the anisotropy parameter the system undergoes a quantum phase transition into a spin-glass state. We do not find any signature of such a transition in the generalized Curie temperature for both inand out-of-plane susceptibility.

The third row of Table I describes a Dirac-RKKY model of magnetic adatoms at the centers of carbon hexagons in graphene. ${ }^{20}$ The quenched parameter $\xi$ labels the three distinct positions of the atom in the $\sqrt{3} \times \sqrt{3}$ superlattice formed by the commensurate Friedel oscillations induced by intervalley scattering. ${ }^{21}$ In this case ferromagnetic ordering is replaced by a staggered state with the order parameter

$$
\Phi=\sum_{i}\left[s_{i}^{x} \cos \left(2 \pi \xi_{i} / 3\right)+s_{i}^{y} \sin \left(2 \pi \xi_{i} / 3\right)\right] .
$$

For graphene decorated with Co atoms one can estimate the Curie temperature $\Theta$ basing on recent density functional theory $^{23}$ and Kondo measurements. ${ }^{24}$ Using the electronimpurity exchange coupling $J a^{3} \sim 1 \mathrm{eV}^{23}$ (here $a$ is the lattice constant) the ordering transition should be expected at roughly $1 \mathrm{~K}$ at $0.1 \%$ coverage of graphene by adatoms. Note that this is a very crude estimate and the actual value of the constant $J$ is to be determined from a comparison of the susceptibility curve with the experiment.

Together with the detailed analysis of the Ising system these examples demonstrate how the proposed generic hightemperature expansion, Eqs. (1), (6), and (7), can be used to describe the magnetic properties of disordered Dirac-RKKY systems.

\section{ACKNOWLEDGMENTS}

We thank I. Aleiner, B. Altshuler, and M. Mezard for helpful discussions. This work was supported by the European Research Council through the Starting Grant "NEDFOQ" and the Advanced Grant "Graphene and Beyond", by the Engineering and Physical Sciences Research Council through the Science and Innovation Award, and by the Royal Society.
${ }^{1}$ M. A. Ruderman and C. Kittel, Phys. Rev. 96, 99 (1954); T. Kasuya, Prog. Theor. Phys. 16, 45 (1956); K. Yosida, Phys. Rev. 106, 893 (1957).

${ }^{2}$ D. I. Golosov and M. I. Kaganov, J. Phys.: Condens. Matter 5, 1481 (1993).

${ }^{3}$ K. S. Novoselov et al., Science 306, 666 (2004).

${ }^{4}$ A. H. Castro Neto, F. Guinea, N. M. R. Peres, K. S. Novoselov, and

A. K. Geim, Rev. Mod. Phys. 81, 109 (2009).

${ }^{5}$ D. Hsieh et al., Nature (London) 452, 970 (2008).

${ }^{6}$ M. Z. Hasan and C. L. Kane, Rev. Mod. Phys. 82, 3045 (2010).

${ }^{7}$ B. Aufray et al., Appl. Phys. Lett. 96, 183102 (2010); B. Lalmi et al., ibid. 97, 223109 (2010).

${ }^{8}$ Y. L. Chen et al., Science 329, 659 (2010).

${ }^{9}$ L. A. Wray et al., Nature Physics 7, 32 (2011).

${ }^{10}$ V. V. Cheianov, O. Syljuasen, B. L. Altshuler, and V. Fal'ko, Europhys. Lett. 89, 56003 (2010); V. V. Cheianov, V. Fal'ko,
O. Suljuasen, and B. L. Altshuler, Solid State Commun. 149, 1499 (2009); V. V. Cheianov, O. Syljuasen, B. L. Altshuler, and V. Fal'ko, Phys. Rev. B 80, 233409 (2009).

${ }^{11}$ D. A. Abanin and D. A. Pesin, Phys. Rev. Lett. 106, 136802 (2011).

${ }^{12}$ L. Brey, H. A. Fertig, S. Das Sarma, Phys. Rev. Lett. 99, 116802 (2007); S. Saremi, Phys. Rev. B 76, 184430 (2007).

${ }^{13}$ G. A. Baker, Jr., and P. Graves-Morris, Padé Approximants (Cambridge University Press, Cambridge, 1996).

${ }^{14}$ M. A. H. Vozmediano, M. P. Lopez-Sancho, T. Stauber, and F. Guinea, Phys. Rev. B 72, 155121 (2005); V. V. Cheianov and V. I. Fal'ko, Phys. Rev. Lett. 97, 226801 (2006); V. K. Dugaev, V. I. Litvinov, and J. Barnas, Phys. Rev. B 74, 224438 (2006).

${ }^{15}$ E. Kogan, Phys. Rev. B 84, 115119 (2011).

${ }^{16}$ There may be more than one equilibrium position of an adatom in the unit cell of the host. For example, an adatom binding covalently to a carbon atom in graphene will have to chose between the A and B sublattices. 
${ }^{17}$ N. Prokof'ev and B. Svistunov, Phys. Rev. Lett. 87, 160601 (2001).

${ }^{18}$ A. F. Albuquerque et al., J. Magn. Magn. Mater. 310, 1187 (2007).

${ }^{19}$ T. G. Rappoport, B. Uchoa, A. H. Castro Neto, Phys. Rev. B 80, 245408 (2009).

${ }^{20} \mathrm{Yu}$. Sherkunov, V. Fal'ko, and V. Cheianov (unpublished).

${ }^{21}$ It is interesting to note that, apart from this interaction, symmetry also admits antiferromagnetic coupling between any two impurities.
However, such coupling was found ${ }^{22}$ to decay much faster than $1 / r^{3}$; therefore it can be neglected in dilute systems.

${ }^{22}$ Bruno Uchoa, T. G. Rappoport, and A. H. Castro Neto, Phys. Rev. Lett. 106, 016801 (2011).

${ }^{23}$ T. O. Wehling, A. V. Balatsky, M. I. Katsnelson, A. I. Lichtenstein, and A. Rosch, Phys. Rev. B 81, 115427 (2010)

${ }^{24}$ L. S. Mattos, C. R. Moon, M. W. Sprinkle, C. Berger, K. Sengupta, A. V. Balatsky, W. A. de Heer, and H. C. Manoharan (submitted to Nature). 